\title{
Laboratory and Field Performance of Five Cost-Effective Commercial Light Traps for Capturing Mosquitoes In China
}

\section{Run Huang}

Wuhan University School of Basic Medical Sciences https://orcid.org/0000-0002-0580-3350

\author{
Hongyun Song \\ Ministry of Agriculture of the People's Republic of China \\ Qian Fang \\ Wuhan University \\ Junping Qian \\ Wuhan University \\ Yaodan Zhang \\ Wuhan University \\ Hong Jiang ( $\nabla$ jiangh@whu.edu.cn ) \\ School of Basic Medical Sciences, Wuhan University
}

\section{Research}

Keywords: Mosquito traps, BG-trap, UV wavelength, Fan speed, Aedes albopictus, Culex quinquefasciatus, Anopheles sinensis

Posted Date: December 28th, 2020

DOI: https://doi.org/10.21203/rs.3.rs-133329/v1

License: () (1) This work is licensed under a Creative Commons Attribution 4.0 International License. Read Full License 


\section{Abstract}

Background

Mosquito traps for household use are popular because they are small, cost-effective, user friendly, and environmentally friendly. At present, there are many variations and specifications of mosquito traps intended for household use on the market. Their labels claim they are powerful, but research and evaluation of their claims is lacking.

Methods

This article tested the key parameters, the laboratory capture rate, and the greenhouse field capture rate of 5 popular mosquito traps intended for household use,and compared them with the BG-trap, used by professionals to monitor mosquitoes in the field.

Results

The study found that the wavelength of 395-400 nm had a better capture rate for Culex quinquefasciatus. In the laboratory experiment, the capture rate was between $34.7 \%-65.0 \%$. The analysis showed that the total radiance, fan speed, and design of the air guide of the traps are important factors that affect the mosquito catch rate. Field tests in the greenhouse found that the 5 mosquito traps had low catch rates for Aedes albopictus. The average percentage of $C x$. quinquefasciatus, Ae. albopictus, Anopheles Sinensis, and other flying insects captured every night was $51.76 \%, 25.29 \%, 14.12 \%$, and $8.82 \%$. There was no significant difference in the capture rate of Ae. albopictus by the 5 mosquito traps in the greenhouse, while the mosquito species captured during the same period by the human landing catch method were all Ae. albopictus, suggesting that the dominant species of mosquitoes in the greenhouse was Ae. albopictus. The comparison experiment of mosquito trap 5, with the highest capture rate in the laboratory simulation and greenhouse site, and the BG-trap in the morning, afternoon, and night showed that the capture rate of the BG-trap on Ae. albopictus and $C x$. quinquefasciatus was higher than that of mosquito trap 5. Combined with the results of the human landing catch method during the same period, it showed that the BG-trap can more accurately reflect the composition of the mosquito community.

\section{Conclusions}

According to this study, it is suggested that the current $395-400 \mathrm{~nm}$ wavelength mosquito traps are not suitable for mosquito control measures in the domestic indoor environment where Ae. albopictus is the dominant species. The mosquito traps intended for household use can be improved by increasing the fan speed and optimizing the air guide. With a higher catch rate, the BG-trap is more suitable for mosquito monitoring than the UV-trap.

\section{Introduction}

Mosquito-borne diseases such as malaria, dengue fever, Chikungunya fever, and Zika are major threats to global health, especially dengue fever, which has increased 30-fold in the past 50 years(Huang, et al. 2019; Jelinek 2018; Lee, et al. 2018; Wilder-Smith, et al. 2019). Of the more than 50 mosquito-borne diseases known, vaccines are available only for epidemic encephalitis and yellow fever. The only way to curb other mosquito-borne diseases is mosquito control(Baldacchino, et al. 2015; de Thoisy, et al. 2020; Idoko, et al. 2020; Oliveira, et al. 2019; Trovato, et al. 2020). During mosquito season or a mosquito-borne disease epidemic, it is necessary not only to implement integrated mosquito control in the external environment, but also to control mosquitoes and prevent bites in residents' homes.

In malaria-endemic Africa, indoor control methods for the malaria vector Anopheles mosquitoes are mainly indoor residual spraying (IRS), which usually requires implementation by professional pest control personnel(Bouckenooghe, et al. 2019; Hamre, et al. 2020; Hien, et al. 2020; Kakilla, et al. 2020; Lees, et al. 2020). Mosquito incense and aerosols are traditional mosquito control methods in the home environment in dengue-endemic Southeast Asia and southern China. Due to the social attention to environmental protection and health concern, the number of families who have begun to adopt non-chemical mosquito control methods is increasing. One of these methods is the use of a mosquito trap.

Mosquito traps intended for household use are based on light mosquito trapping techniques for mosquito surveillance, such as the ultraviolet light trap for mosquito surveillance issued by the Centers for Disease Control and Prevention (CDC) (Holderman, et al. 2018; Li, et al. 2016; Silva, et al. 2019; Sriwichai, et al. 2015). Mosquito traps for household use are popular because they are small, costeffective, user friendly, and environmentally friendly. The design principle of this kind of product is the same as that used by 
professionals. The light source used is an ordinary ultraviolet light or light-emitting diode (LED). The wavelength range of the ultraviolet light is $320 \mathrm{~nm}-400 \mathrm{~nm}$, and a fan is set to form a guiding airflow, which draws the mosquitoes into the mosquito collection device, where they are trapped.

At present, there are many variations and specifications of mosquito traps intended for household use on the market. Their labels claim they are powerful, but research and evaluation of their claims is lacking. For that reason, we selected five popular mosquito traps costing 30 USD or less and evaluated their mosquito control performance in the home environment. We paid attention especially to how well they were made, and their mosquito capturing performance in the laboratory and in the field to provide valuable information for the control of mosquito-borne diseases by mosquito traps for household use.

\section{Material And Methods}

\section{Description of the Five Light Traps and Quality Check Source of mosquito trap}

For this study, we purchased ultraviolet mosquito traps intended for household use from a well-known eCommerce store in China. We selected five of the most popular variations of mosquito traps with a price not exceeding 30 USD for research and evaluation. The product parameters are shown in Table 1.

Table 1

Parameters of five household mosquito traps used in the experiment

\begin{tabular}{|llllll|}
\hline Product No. & Product Model & Power(W) & Power Supply & Net Weight(g) & Product Dimensions(mm) \\
\hline Trap 1 & DH-03S & 5 & DC5V & 430 & $120 \star 174$ \\
\hline Trap 2 & MY-100 & $5-0.5$ & DC5V-0.5A & 370 & $137 \star 225$ \\
\hline Trap 3 & KLY-188 & 5 & $220 V / 50 H Z$ & 200 & $120 \star 215$ \\
\hline Trap 4 & N/A & 5 & DV 5V-1A & 310 & $120 \star 220$ \\
\hline Trap 5 & N/A & 4 & $220 \mathrm{~V}$ & 500 & $170 * 340$ \\
\hline
\end{tabular}

\section{Detection of radiation wavelength of mosquito traps}

The radiation wavelength detection of the mosquito traps' UV light was conducted in the Mosquito Trap Quality Monitoring Laboratory of Zhongshan Protostar Optoelectronic Co., Ltd. The test method conformed to the inspection method for appliances with ultraviolet radiation lamps stipulated in Article 32 of the Chinese National Standard Household and Similar Electrical Appliances-SafetyParticular Requirements for Insect Killers(Institute, et al. 2008). The appliances were supplied with rated voltage and operated under normal working conditions. The test equipment used was the PMS-80 ultraviolet (UV)-visible (VIS)-near infrared (NIR) spectroscopy analysis system, which measures radiation at $1 \mathrm{~m}$. The maximum radiation should be recorded when the measuring instrument is placed.

According to the specific operation process of the spectrometric testing instrument, the five variations of the mosquito traps were determined in order of number, and the data obtained after the test were analyzed. The total effective radiation was calculated by the following formula.

$$
\mathrm{E}=\sum_{250 \mathrm{~nm}}^{400 n m} S_{\lambda} E_{\lambda} \Delta_{\lambda}
$$

Where:

E--Effective Radiation; 
$\mathrm{S}_{\lambda}--$ Relative Spectral Weight Factor;

$E_{\lambda}--$ Spectral Irradiance $\left(W / m^{2} n m\right)$

$\Delta_{\lambda}--$ Bandwidth $(\mathrm{nm})$

When measuring spectral irradiance, the radiation required a stable light source. The effective radiation of each wavelength is calculated as the spectrum according to the ultraviolet (UV) of the spectral weight factor of different wavelengths. The total effective radiation (E) should not exceed $1 \mathrm{~mW} / \mathrm{m}^{2}$.

\section{Ultraviolet mosquito trap air suction fan speed determination}

The test was carried out following the stipulations of the Chinese National Standard A. C. Fans and Regulators(Institute, et al. 2018), and the test equipment used was the Hima-split anemometer AS8336. During the test, only the anemometer can be placed in front of the outlet of the fan. In the middle of the test, the tester can stay at the inlet side. The tester is only allowed to enter the fan outlet area when they need to control the speed and read the data. The tester should take minimum time to record the data and control the fan speed. The measurement begins about $20 \mathrm{~mm}$ from the air outlet side. For a more accurate result, the fan is sectioned into 4 quadrants (points). The anemometer is used to test the outgoing wind speed of each quadrant of the fan. Afterward, the value indicated by the anemometer is divided by the sampling time of the anemometer at that quadrant to measure the wind speed $(\mathrm{m} / \mathrm{s})$. The time used in measuring wind speed should not be less than 1 min.

\section{Laboratory Test}

\section{Laboratory simulation field test experiment}

A simulated field test was conducted in the Pesticide Evaluation Laboratory of Ningbo Yuying Vector Biocontrol Co., Ltd. in Zhejiang Province from May 25, 2020, to July 10, 2020. The test method followed the stipulations of the Chinese National Standard Laboratory Efficacy Test Methods and Criterion of Public Health Equipment-Electronic Trap for Mosquitoes and Flies(Prevention, et al. 2011). The glass test room was $3 \mathrm{~m}$ long, $3 \mathrm{~m}$ wide, and $3 \mathrm{~m}$ high, approximating a square room with a volume of $27 \mathrm{~m}^{3}$. The test insect was $C X$. quinquefasciatus. For the insecticide-sensitive strain of $C x$. quinquefasciatus bred in this laboratory, female adult mosquitoes 3-5 days after emergence without blood suction were selected. The test conditions comprised temperature $26{ }^{\circ} \mathrm{C} \pm 1{ }^{\circ} \mathrm{C}$ and relative humidity $65 \% \pm 10 \%$. The experiment started at $5 \mathrm{p}$.m. The mosquito trap to be tested was placed in the center of the test room and the light source was set $1.5 \mathrm{~m}$ away from the ground. Next, we released 100 mosquitoes into the test room, closed the doors and windows, and turned on the power supply to the mosquito trap after the test insects resumed their normal activities. At 8 a.m. on the second day, we cut off the power supply and wrapped the mosquito trap in a silk yarn cage to prevent the mosquitoes from escaping. Afterward, we extracted the mosquito trap's collection device to check the number of test mosquitoes captured to calculate the capture rate.

Capture rate $=$ number of mosquitoes captured/number of mosquitoes released in the room $\times 100 \%$

The test was repeated three times. The blank control was tested by turning on the fan, but not the light.

\section{Field Test}

The greenhouse field capture test was performed for the first time in November 2019 and for the second time in mid-July 2020. The site used for the field tests was the greenhouse of Guangxi Pastoral Biochemical Co., Ltd. in Nanning City, Guangxi Zhuang Autonomous Region. Geographically, the test site is $108.26^{\circ}$ longitude, $22.86^{\circ}$ latitude, and $77 \mathrm{~m}$ above sea level. It has a humid subtropical monsoon climate, with an annual average temperature of $20^{\circ} \mathrm{C}-29^{\circ} \mathrm{C}$ and annual average precipitation of $1304.2 \mathrm{~mm}$. The greenhouse is $21 \mathrm{~m}$ long, $14 \mathrm{~m}$ wide, and $5 \mathrm{~m}$ high. The average daily temperature and humidity in the greenhouse are $30{ }^{\circ} \mathrm{C}$ and $80 \%$, respectively. Corn, eggplants, rice, peppers, and other crops are planted all year round.

The test method followed the mosquito trap method and the human landing catch method stipulated in the China National Standard for Vector Biodensity Monitoring Method-Mosquitoes (Department of Analytical Microbiology 2009). In the mosquito trap method, we placed the mosquito traps in a sheltered area away from any interfering light source. The mosquito traps to be tested were placed more than $15 \mathrm{~m}$ apart, with plants between them to prevent light from any lamp from interfering with any other lamp. The light source of the mosquito trap was placed $1.5 \mathrm{~m}$ away from the ground. One hour before sunset, we turned on the mosquito traps to start the test. The power remained on until 1 hour after sunrise the next day. After turning off the lamp, we wrapped the mosquito traps with a silk yarn

Page $4 / 13$ 
cage to prevent the captured mosquitoes from escaping. Then we counted and categorized the number and species of female mosquitoes captured. This test was carried out in two phases, each of which was repeated three times with the trap in a fixed position.

The human landing catch test was performed 30 minutes before the test of the mosquito traps. First, the monitors exposed one leg and remained stationary. Second, the species and number of mosquitoes that landed on the leg and were captured by the electric mosquito trap within 30 minutes were recorded. Lastly, the time, location, temperature, humidity, and wind speed at the beginning and end of the human landing catch test were recorded.

\section{Comparative study of capture efficacy of the mosquito trap for household use and the BG-trap}

The BG-trap (Biogents AG, Regensburg, Germany), used by professionals, is a foldable mosquito monitoring device, $36 \mathrm{~cm}$ in diameter and $40 \mathrm{~cm}$ in height. It's designed with a black tube in the middle of the plastic surface plate and a small fan under the tube connected to a removable mosquito collection device. Bait called BG-Sweetscent is placed in the container, which releases a mixture of lactic acid, ammonia, and hexanoic acid that mimics the odor on the surface of human skin to attract mosquitoes into the pipeline. Lastly, the fan airflow draws the attracted mosquitoes into the mosquito collection device. Unlike the five popular mosquito traps tested, it does not use a light to attract mosquitoes. It uses scent instead(Bhalala and Arias 2009; Ponlawat, et al. 2017).

Of the five mosquito traps, we selected the one with the highest capture rate for comparative testing with the BG-trap. The test was conducted in the same greenhouse as the previous experiment. Both mosquito control devices were placed on a shelf $1.5 \mathrm{~m}$ away from the ground. They were placed $15 \mathrm{~m}$ apart from each other. The tests were conducted three times per day. The testing periods for each day were as follows: 1) 9:00 A.M. to 11:00 A.M. 2) 2:00 P.M. to 4:00 P.M. 3) 6:00 P.M. to second day morning 8:00 A.M. After each test, the positions of the two mosquito traps were exchanged. Each mosquito control device was tested three times at each location and at each period.

\section{Species Identification and Statistical Analysis}

Morphological identification of mosquitoes captured, including mosquito species and genders, was performed using an anatomical microscope and the capture performance of the five mosquito traps was evaluated. All statistical analyses were performed using RStudio (Version 1.2.5001, 64bit) and R (version 3.4.1, 64bit) backends. In terms of statistical significance level, * means $P<0.05$, ** means $\mathrm{P}<0.01$, *** means $\mathrm{P}<0.001$.

\section{Results}

\section{Product Quality}

The ultraviolet wavelengths of the five mosquito traps were measured by a PMS-80 ultraviolet (UV)-visible (VIS)-near infrared (NIR) spectroscopy analysis system. The results are shown in Table 2. According to the Chinese National Standard Household and Similar Electrical Appliances-Safety-Particular Requirements for Insect Killers (Institute, et al. 2008), mosquito traps exceeding $1 \mathrm{~mW} / \mathrm{m}^{2}$ total effective radiation exceed that which is allowed and are deemed unqualified. Therefore, mosquito trap 5 is judged to be unqualified, because its total effective radiation is $2.1980 \mathrm{~mW} / \mathrm{m}^{2}$, which exceeds the standard allowance. Whereas the remaining 4 traps are qualified.

Table 2

UV Wavelength Determination and Fan Speed Results of Five Household Mosquito Traps

\begin{tabular}{|llll|}
\hline Product No. & UV wavelength(nm) & Effective Radiation $\left(\mathrm{mW} / \mathbf{m}^{2}\right)$ & Fan Speed(m/s) \\
\hline Trap 1 & 400 & 0.1300 & 1.53 \\
\hline Trap 2 & 400 & 0.3902 & 1.32 \\
\hline Trap 3 & 395 & 0.0874 & 1.01 \\
\hline Trap 4 & 400 & 0.3029 & 1.15 \\
\hline Trap 5 & 395 & 2.1980 & 2.10 \\
\hline
\end{tabular}


The fan speed test results of the suction fan of the traps are shown in Table 2 . The average fan speed of mosquito trap 5 is $2.10 \mathrm{~m} / \mathrm{s}$, which is the highest among all the traps.

\section{Laboratory Tests}

The results of the mosquito capture rate test in the laboratory are shown in Table 3 . The Shapiro-Wilk test was used to verify that the data were normally distributed. The results of variance analysis and the Tukey test showed that there were significant differences in the trapping rate of the five mosquito traps $(\mathrm{P}<0.001)$. The capture rate of mosquito trap 5 and 1 exceeded $50 \%$, which was significantly higher than that of the other three mosquito traps.

Table 3

Laboratory Test Capture Rate of Five Household Mosquito Traps

\section{Field Tests}

\begin{tabular}{|llll|}
\hline Product No. & Total release number & Re-capturing number & Capturing Rate (\%) \\
\hline Trap 1 & 300 & 179 & 59.7 \\
\hline Trap 2 & 300 & 136 & 45.3 \\
\hline Trap 3 & 300 & 121 & 40.3 \\
\hline Trap 4 & 300 & 104 & 34.7 \\
\hline Trap 5 & 300 & 195 & 65.0 \\
\hline
\end{tabular}

In the first phase of the greenhouse field test, 170 specimens were collected from the five mosquito traps; 143 (84.12\%) of the total specimens were female mosquitoes. The mosquito species captured most was the $C x$. quinquefasciatus, 88 of which were captured, comprising $51.76 \%$ of the total number of mosquitoes captured. This was followed by Ae. albopictus, 43 of which were captured, comprising $25.29 \%$ of the total captured and An. sinensis, 24 of which were captured, comprising $14.12 \%$ of the total captured. The above three species of mosquito are the prevalent species in the urban environment of China, and thus, were the most captured mosquito species in our test (Table 4). The average number of mosquitoes captured per test round ( 30 min) was $2.33+0.82$ by the human landing catch method, and all the captured mosquitoes were female Ae. albopictus.

Table 4

Comparison of five mosquito traps in field settings

\begin{tabular}{|c|c|c|c|c|c|c|c|c|c|c|c|c|}
\hline mosquito & trap 1 & & trap 2 & & trap 3 & & trap 4 & & trap 5 & & Total & $\%$ \\
\hline & $\begin{array}{l}\text { Mean } \pm \\
\text { SE }\end{array}$ & $\mathrm{N}$ & $\begin{array}{l}\text { Mean } \pm \\
\text { SE }\end{array}$ & $\mathrm{N}$ & $\begin{array}{l}\text { Mean } \pm \\
\text { SE }\end{array}$ & $\mathrm{N}$ & $\begin{array}{l}\text { Mean } \pm \\
\text { SE }\end{array}$ & $\mathrm{N}$ & $\begin{array}{l}\text { Mean } \pm \\
\text { SE }\end{array}$ & $\mathrm{N}$ & & \\
\hline Ae. albopictus & $\begin{array}{l}1.00 \pm \\
0.21\end{array}$ & 6 & $\begin{array}{l}1.33 \pm \\
0.33\end{array}$ & 8 & $\begin{array}{l}1.17 \pm \\
0.40\end{array}$ & 7 & $\begin{array}{l}1.00 \pm \\
0.37\end{array}$ & 6 & $\begin{array}{l}2.67 \pm \\
0.61\end{array}$ & 16 & 43 & 25.29 \\
\hline Cx. quinquefasciatus & $\begin{array}{l}4.83 \pm \\
0.70\end{array}$ & 29 & $\begin{array}{l}1.83 \pm \\
0.31\end{array}$ & 11 & $\begin{array}{l}1.50 \pm \\
0.42\end{array}$ & 9 & $\begin{array}{l}0.67 \pm \\
0.21\end{array}$ & 4 & $\begin{array}{l}5.83 \pm \\
0.60\end{array}$ & 35 & 88 & 51.76 \\
\hline An. sinensis & $\begin{array}{l}0.84 \pm \\
0.40\end{array}$ & 5 & $\begin{array}{l}0.50 \pm \\
0.22\end{array}$ & 3 & $\begin{array}{l}0.33 \pm \\
0.21\end{array}$ & 2 & $\begin{array}{l}1.00 \pm \\
0.36\end{array}$ & 6 & $\begin{array}{l}1.33 \pm \\
0.42\end{array}$ & 8 & 24 & 14.12 \\
\hline others & $\begin{array}{l}0.67 \pm \\
0.33\end{array}$ & 4 & $\begin{array}{l}0.33 \pm \\
0.21\end{array}$ & 2 & $\begin{array}{l}0.50 \pm \\
0.22\end{array}$ & 3 & $\begin{array}{l}0.17 \pm \\
0.17\end{array}$ & 1 & $\begin{array}{l}0.83 \pm \\
0.40\end{array}$ & 5 & 15 & 8.82 \\
\hline $\begin{array}{l}\text { Total number of } \\
\text { individuals }\end{array}$ & 44 & & 24 & & 21 & & 17 & & 64 & & 170 & \\
\hline Total number of female & 38 & & 20 & & 16 & & 15 & & 54 & & 143 & 84.12 \\
\hline
\end{tabular}


Table 5

Capture performance of BG vs UV trap in field settings

\begin{tabular}{|c|c|c|c|c|c|c|c|c|c|c|c|c|c|c|}
\hline \multirow[t]{3}{*}{ mosquito } & \multicolumn{6}{|c|}{ BG-trap } & \multicolumn{6}{|c|}{ UVtrap } & \multirow[t]{3}{*}{ Total } & \multirow[t]{3}{*}{$\%$} \\
\hline & \multicolumn{2}{|c|}{ morning } & \multicolumn{2}{|c|}{ afternoon } & \multicolumn{2}{|c|}{ evening } & \multicolumn{2}{|c|}{ morning } & \multicolumn{2}{|c|}{ afternoon } & \multicolumn{2}{|l|}{ evening } & & \\
\hline & $\begin{array}{l}\text { Mean } \\
\pm S E\end{array}$ & $\mathrm{~N}$ & $\begin{array}{l}\text { Mean } \\
\pm S E\end{array}$ & $\mathrm{~N}$ & $\begin{array}{l}\text { Mean } \\
\pm S E\end{array}$ & $\mathrm{~N}$ & $\begin{array}{l}\text { Mean } \\
\pm S E\end{array}$ & $N$ & $\begin{array}{l}\text { Mean } \\
\pm S E\end{array}$ & $\mathrm{~N}$ & $\begin{array}{l}\text { Mean } \\
\pm S E\end{array}$ & $\mathrm{~N}$ & & \\
\hline Ae. albopictus & $\begin{array}{l}4.83 \\
\pm 0.91\end{array}$ & 29 & $\begin{array}{l}5.17 \\
\pm 0.75\end{array}$ & 31 & $\begin{array}{l}37.67 \\
\pm 1.67\end{array}$ & 226 & $\begin{array}{l}0.67 \\
\pm 0.33\end{array}$ & 4 & $\begin{array}{l}0.17 \\
\pm 0.17\end{array}$ & 1 & $\begin{array}{l}7.00 \\
\pm 1.15\end{array}$ & 42 & 333 & 72.87 \\
\hline $\begin{array}{l}\text { Cx. } \\
\text { quinquefasciatus }\end{array}$ & $\begin{array}{l}3.00 \\
\pm 1.18\end{array}$ & 18 & $\begin{array}{l}2.00 \\
\pm 0.37\end{array}$ & 12 & $\begin{array}{l}6.33 \\
\pm 0.82\end{array}$ & 38 & $\begin{array}{l}0.17 \\
\pm 0.17\end{array}$ & 1 & $\begin{array}{l}0.33 \\
\pm 0.21\end{array}$ & 2 & $\begin{array}{l}3.33 \\
\pm 0.92\end{array}$ & 20 & 91 & 19.91 \\
\hline An. sinensis & $\begin{array}{l}1.17 \\
\pm 0.48\end{array}$ & 7 & $\begin{array}{l}0.67 \\
\pm 0.33\end{array}$ & 4 & $\begin{array}{l}2.83 \\
\pm 0.60\end{array}$ & 17 & $\begin{array}{l}0.17 \\
\pm 0.17\end{array}$ & 1 & $\begin{array}{l}0.17 \\
\pm 0.17\end{array}$ & 1 & $\begin{array}{l}0.50 \\
\pm 0.34\end{array}$ & 3 & 33 & 7.22 \\
\hline $\begin{array}{l}\text { Total number of } \\
\text { individuals }\end{array}$ & 382 & & & & & & 75 & & & & & & 457 & \\
\hline $\begin{array}{l}\text { Total number of } \\
\text { females }\end{array}$ & 318 & & & & & & 60 & & & & & & 378 & 82.71 \\
\hline
\end{tabular}

After the Shapiro-Wilk test was performed to confirm that the data were not a normal distribution, the Kruskal-Wallis test was performed. The results are shown in Fig. 1

The results showed that mosquito trap 5 caught the most Ae. albopictus on average every night $(2.67 \pm 0.61)$. However, there was no significant difference in the average number of mosquitoes captured per night among the five traps $(P=0.17)$. The results are shown in Fig. 1A.

The results of the number of $C x$. quinquefasciatus captured showed that there were significant differences among the five mosquito traps $(P<0.001)$. The average number of mosquitoes captured per night of trap 5 was about 8.7 times that of trap $4(P<0.01)$, about 3.9 times that of trap $3(P=0.031)$, and about 7.2 times that of trap $1(P=0.0065)$. The results are shown in Fig. 1B.

In terms of the number of An. sinensis captured, only trap 5 captured more than 1 of this species, on average, and there was no significant difference among the five mosquito traps $(P=0.315)$. The results are shown in Fig. $1 \mathrm{C}$.

The Kruskal-Wallis test result showed that there were significant differences among the five mosquito traps in the average total number of mosquitoes captured per night $(P<0.001)$. The average number of mosquito captured per night of trap 5 was about 3.7 times that of trap $4(P<0.001)$, about 3.0 times that of trap $3(P<0.01)$, about 2.66 times that of trap $2(P=0.023)$, and about 1.8 times that of trap 1 $(P=0.039)$. The mosquito capture performance of trap 5 was the best among the five traps, followed by trap 1 . The results are shown in Fig. 1D.

In the first phase of the test in the greenhouse, the capture performance of trap 5 was the best. Thus, we decided to compare the performance of trap 5 with that of the BG-trap. We renamed trap 5 as UV-trap and compared it with the BG-trap in the greenhouse18 times. After the test, a total of 457 specimens were collected.

The second phase of the test was divided into three periods: morning, afternoon, and evening. A total of 457 mosquitoes were captured, 378 of which were female (82.7\%). The largest ratio of mosquito species trapped was Ae. albopictus, of which 333 were captured, accounting for more than half of the total number (72.9\%), followed by 91 Cx. quinquefasciatus (19.9\%) and 33 An. sinensis (7.2\%). Next, we analysed the conditions under which the mosquitoes were captured in different periods. The results are shown in Fig. 2. As for the result of the human landing catch method, the average number of captured mosquitoes per time ( $30 \mathrm{~min})$ in the morning, afternoon, and evening were $1.67 \pm 0.33,1.83 \pm 0.17,3.00 \pm 0.37$, respectively. All the mosquitoes captured in the three periods were Ae. albopictus.

In the morning (Fig. 2A), the performance of the BG-trap in capturing Ae. albopictus and Cx. quinquefasciatus was significantly higher than that of the UV-trap. The number of Ae. albopictus captured by the BG-trap ( $4.83 \pm 0.91)$ was 7.2 times higher than that of the UV- 
$\operatorname{trap}(0.67 \pm 0.33)(P<0.01)$. The number of $C x$. quinquefasciatus captured by the BG-trap $(3.00 \pm 1.18)$ was 17.6 times higher than that of the UV-trap $(0.17 \pm 0.17)(P<0.05)$.

In the afternoon (Fig. 2B), the performance of the BG-trap in capturing Ae. albopictus and Cx. quinquefasciatus was significantly higher than that of the UV-trap. The number of Ae. albopictus captured by the BG-trap ( $5.17 \pm 0.75)$ was 30.4 times higher than that captured by the UV-trap $(0.17 \pm 0.17)(P<0.001)$. The number of $C X$. quinquefasciatus captured by the BG-trap $(2.00 \pm 0.37)$ was 6.1 times higher than that captured by the UV-trap $(0.33 \pm 0.21)(P<0.01)$.

In the evening (Fig. 2C), the performance of the BG-trap in capturing the three species of mosquitoes was significantly higher than that of the UV-trap. The number of Ae. albopictus captured by the BG-trap ( $37.67 \pm 1.67)$ was 5.2 times higher than that captured by the UVtrap $(7.00 \pm 1.15)(P<0.001)$, while the number of $C x$. quinquefasciatus captured by the BG-trap $(6.33 \pm 0.82)$ was 1.9 times higher than that captured by the UV-trap $(3.33 \pm 0.92)(P<0.05)$. Lastly, the number of $A n$. sinensis captured by the BG-trap $(2.83 \pm 0.60)$ was 5.7 times higher than that captured by the UV-trap $(0.50 \pm 0.34)(P<0.01)$.

Further, we compared the total number of mosquitoes captured per hour in each period and obtained the result shown in Fig. 2D. The average number of mosquitoes captured per hour by the BG-trap $(3.96 \pm 1.03)$ was higher than that captured by the UV-trap $(0.57 \pm$ $0.54)$, and the difference was significant $(t=12.381, d f=25.78, P<0.001)$.

\section{Discussion}

In this study, the ultraviolet light of all five household mosquito traps had a wavelength range of $390 \mathrm{~nm}-400 \mathrm{~nm}$, which conformed to the standard ultraviolet light range. According to the Pearson correlation coefficient, the correlation coefficient between the ultraviolet light wavelength of the five mosquito traps and the mosquito capture rate in the laboratory was subtle $(P>0.05)$. Therefore, the ultraviolet light wavelength was not a significant factor influencing the difference in the mosquito traps' capture rate.

According to Pearson correlation coefficient analysis on the air suction efficiency of the mosquito traps, the fan speed and capture rate exhibited a linear relationship, which showed that fan speed might be a crucial factor influencing the mosquito capture performance of the traps $(P<0.05)$. Other researchers tested the effect of different fan speeds against the performance of the trap in capturing mosquitoes. The result showed that $1.7 \mathrm{~m} / \mathrm{s}$ was the ideal suction rate to obtain a higher capture rate and lower damage to the captured mosquitoes' bodies(ZHANG Hong-xiang 2002). In our study, mosquito trap 5 had the highest capture rate with an air suction rate of $2 \mathrm{~m} / \mathrm{s}$. And the mosquitoes captured didn't show critical damage to their bodies. Therefore, we believe that whether it is a mosquito trap for household use or a mosquito monitoring trap, the mosquito capture performance can be enhanced by increasing the air suction rate.

Mosquito trap 5 and trap 1 had the highest mosquito capture rates during the laboratory field test. This may be due to their shape and structural design, which were different from the other three mosquito traps. Trap 5 and trap 1 have inclined upward-opening entries, which means they can capture mosquitoes from $360^{\circ}$ around the top, whereas the entries of the other three traps are located at the middle, where the airflow into the entries is parallel and thus there is a smaller capture area. The capture area might be one factor influencing the mosquito capture rate.

In the first phase of the greenhouse field test, the largest ratio of one species of mosquito species captured by the five mosquito traps was $51.76 \%$ and the species captured was $C x$. quinquefasciatus, while the main mosquito species captured by the human landing catch method was that of Ae. albopictus. However, Ae. albopictus was not the main species of mosquito captured by the five mosquito traps and did not reflect the mosquito species composition of the mosquito community in the field. This experiment shows that the ultraviolet trap with a wavelength of $390 \mathrm{~nm}-400 \mathrm{~nm}$ is inefficient in capturing Ae. albopictus and is not suitable for use as a light source for mosquito traps for household use in areas where Ae. albopictus is the dominant species, such as China. It has been reported that the capture rate of a light source with $520 \mathrm{~nm}$ wavelength had a higher capture rate of Ae. albopictus, which deserves further study(Costa-Neta, et al. 2017).

Mosquito trap 5 and trap 1 had a relatively high capture rate of mosquitoes, which was significantly higher than that of the other three traps. Further, the difference was particularly significant in the capturing of $C x$. quinquefasciatus. The total effective radiation of mosquito trap 5 exceeded the standard quite a bit, and its air suction rate was also the largest, which may be the reason for its high capture rate. Mosquito trap 1 achieved high capture efficiency under the premise of product compliance and should be an excellent choice among the five mosquito traps for household use that we evaluated. 
The published comparative test studies of the BG-trap and the UV-trap were conducted in the field, and this experiment evaluated both in the laboratory(Hoshi, et al. 2019; Ponlawat, et al. 2017). The experiment showed that in the greenhouse, the capture rate of Ae. albopictus and $C x$. quinquefasciatus by the BG-trap (used by professionals) in the morning, afternoon, and evening was significantly higher than that by the UV-trap (mosquito trap 5). The capture rate of the BG-trap of Ae. albopictus was higher, which was corroborated by the human landing catch method and reflected the composition of the mosquito community more accurately. In the field of mosquito surveillance, the BG-trap is more objective and accurate than a lamp trap, and it can replace the human landing catch method.

There are few studies on the capture rate of mosquito traps for household use. This study tested the product parameters of five popular mosquito traps, the capture rate in the laboratory and the capture rate in a greenhouse, and obtained preliminary data, which provided research and development ideas for improving the performance of mosquito traps marketed for household use in China.

\section{Declarations}

\section{Acknowledgments}

The authors thank AiMi Academic Services (www.aimieditor.com) for English language editing and review services.

Thanks to Ningbo Yuying Vector Biocontrol Co., Ltd. (www.nbyuying.com) for providing the laboratory test site.

Thanks to Zhongshan Protostar Optoelectronic Co., Ltd. (www.philite.com) for providing the ultraviolet radiation test equipment.

\section{Declaration of Competing Interest}

The authors declare that they have no known competing financial interests or personal relationships that could have appeared to influence the work reported in this paper.

\section{Funding sources}

This research did not receive any specific grant from funding agencies in the public, commercial, or not-for-profit sectors.

\section{Author's contributions}

Hong Jiang conceived and designed the experiments. Run Huang, Hongyun Song, Qian Fang, Junping Qian,Yaodan Zhang performed the experiments. Hong Jiang and Run Huang analyzed the data and wrote the paper. Hong Jiang and Run Huang critically revised the manuscript. All authors read and approved the final version of the submitted manuscript.

\section{Author details}

1 Department of Parasitology, School of Basic Medical Sciences, Wuhan University, Wuhan 430071, Hubei Province, China.

2 Key Laboratory of Pesticides Development and Application Technology, Ministry of Agriculture, P.R.China.

\section{References}

Baldacchino, F., et al.

2015 Control methods against invasive Aedes mosquitoes in Europe: a review. Pest Manag Sci 71(11):1471-85.

Bhalala, H., and J. R. Arias

2009 The Zumba mosquito trap and BG-Sentinel trap: novel surveillance tools for host-seeking mosquitoes. J Am Mosq Control Assoc 25(2):134-9.

Bouckenooghe, A., F. Bailleux, and E. Feroldi

2019 Modeling the long-term persistence of neutralizing antibody in children and toddlers after vaccination with live attenuated Japanese encephalitis chimeric virus vaccine. Hum Vaccin Immunother 15(1):72-79. 
Costa-Neta, B. M., et al.

2017 Light-Emitting Diode (LED) Traps Improve the Light-Trapping of Anopheline Mosquitoes. J Med Entomol 54(6):1699-1703.

de Thoisy, B., et al.

2020 Spatial epidemiology of yellow fever: Identification of determinants of the 2016-2018 epidemics and at-risk areas in Brazil. PLoS Negl Trop Dis 14(10):e0008691.

Department of Analytical Microbiology, Academy of Military Medical Sciences

2009 Surveillance methods for vector density-Mosquito. Pp. 20, Vol. GB/T 23797-2009: General Administration of Quality Supervision, Inspection and Quarantine of the People's Republic of China;Standardization Administration.

Hamre, K. E. S., et al.

2020 A Mass Insecticide-Treated Bed Net Distribution Campaign Reduced Malaria Risk on an Individual but Not Population Level in a Highland Epidemic-Prone Area of Kenya. Am J Trop Med Hyg.

Hien, A. S., et al.

2020 Short Persistence and Vector Susceptibility to Ficam 80WP (bendiocarb active ingredient) During Pilot Application of Indoor Residual Spraying in Burkina Faso, West Africa. J Med Entomol.

Holderman, C. J., et al.

2018 Mosquitoes (Diptera: Culicidae) Collected From Residential Yards and Dog Kennels in Florida Using Two Aspirators, a Sweep Net, or a CDC Trap. J Med Entomol 55(1):230-236.

Hoshi, T., et al.

2019 Field testing of a lightweight, inexpensive, and customisable 3D-printed mosquito light trap in the UK. Sci Rep 9(1):11412.

Huang, Y. S., S. Higgs, and D. L. Vanlandingham

2019 Emergence and re-emergence of mosquito-borne arboviruses. Curr Opin Virol 34:104-109.

Idoko, O. T., et al.

2020 Serological Protection 5-6 Years Post Vaccination Against Yellow Fever in African Infants Vaccinated in Routine Programmes. Front Immunol 11:577751.

Institute, China Household Electric Appliance Research, et al.

2008 Household and similar electrical appliances-Safety-Particular requirements for insect killers. Pp. 16, Vol. GB 4706.76-2008: General Administration of Quality Supervision, Inspection and Quarantine of the People's Republic of China;Standardization Administration.

Institute, China National Electric Apparatus Research, et al.

2018 A.C. electric fans and regulators. Pp. 36, Vol. GB/T 13380-2018: State Administration for Market Regulation;Standardization Administration.

Jelinek, T.

2018 [Mosquito-transmitted infections]. Internist (Berl) 59(1):57-73.

Kakilla, C., et al. 
2020 Malaria vector species composition and entomological indices following indoor residual spraying in regions bordering Lake Victoria, Tanzania. Malar J 19(1):383.

Lee, H., S. Halverson, and N. Ezinwa

2018 Mosquito-Borne Diseases. Prim Care 45(3):393-407.

Lees, R. S., et al.

2020 Tenebenal: a meta-diamide with potential for use as a novel mode of action insecticide for public health. Malar $\mathrm{J} 19(1): 398$.

Li, Y., et al.

2016 Comparative evaluation of the efficiency of the BG-Sentinel trap, CDC light trap and Mosquito-oviposition trap for the surveillance of vector mosquitoes. Parasit Vectors 9(1):446.

Oliveira, A. R. S., et al.

2019 Introduction of the Japanese encephalitis virus (JEV) in the United States - A qualitative risk assessment. Transbound Emerg Dis 66(4):1558-1574.

Ponlawat, A., et al.

2017 Field evaluation of two commercial mosquito traps baited with different attractants and colored lights for malaria vector surveillance in Thailand. Parasit Vectors 10(1):378.

Prevention, Guangdong Provincial Center for Disease Control and, et al.

2011 Laboratory efficacy test methods and criterions of public health equipment-Electronic trap for mosquitoes and flies. Pp. 8, Vol. GB/T 27785-2011: National Health Commission of the People's Republic of China;Standardization Administration.

Silva, F. S., et al.

2019 Field performance of a low cost, simple-to-build, non-motorized light-emitting diode (LED) trap for capturing adult Anopheles mosquitoes (Diptera: Culicidae). Acta Trop 190:9-12.

Sriwichai, P., et al.

2015 Evaluation of CDC light traps for mosquito surveillance in a malaria endemic area on the Thai-Myanmar border. Parasit Vectors 8:636.

Trovato, M., et al.

2020 Viral Emerging Diseases: Challenges in Developing Vaccination Strategies. Front Immunol 11:2130.

Wilder-Smith, A., et al.

2019 Dengue. In Lancet. Pp. 350-363, Vol. 393.

ZHANG Hong-xiang, WANG De-ming, GUAN En-feng, et al

2002 Study on the DW-1Mosquito Trap. Chin J Vector Bio \& Control (06):442-444.

\section{Figures}


A

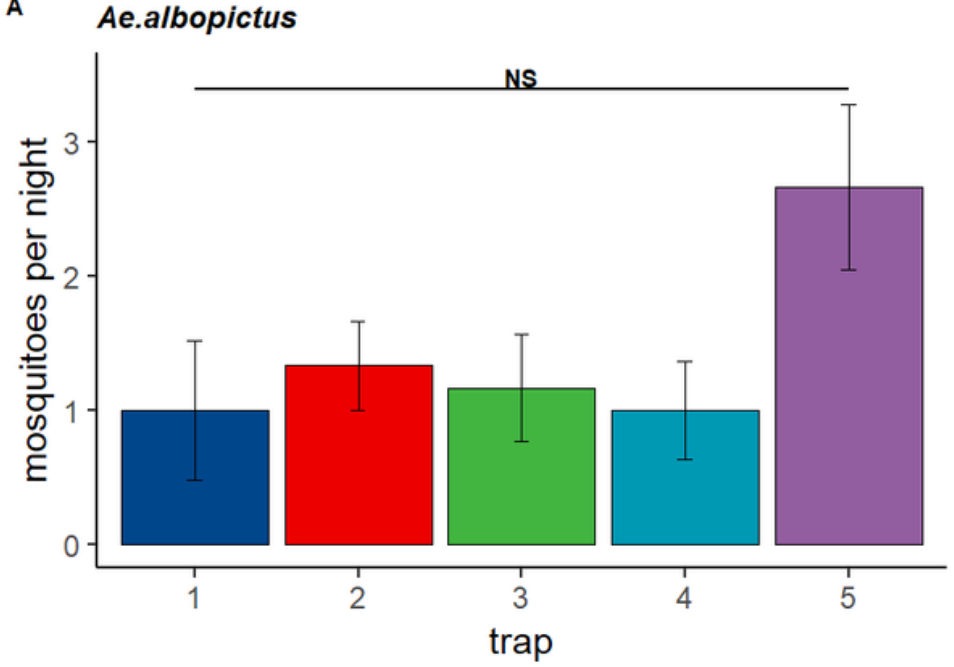

C

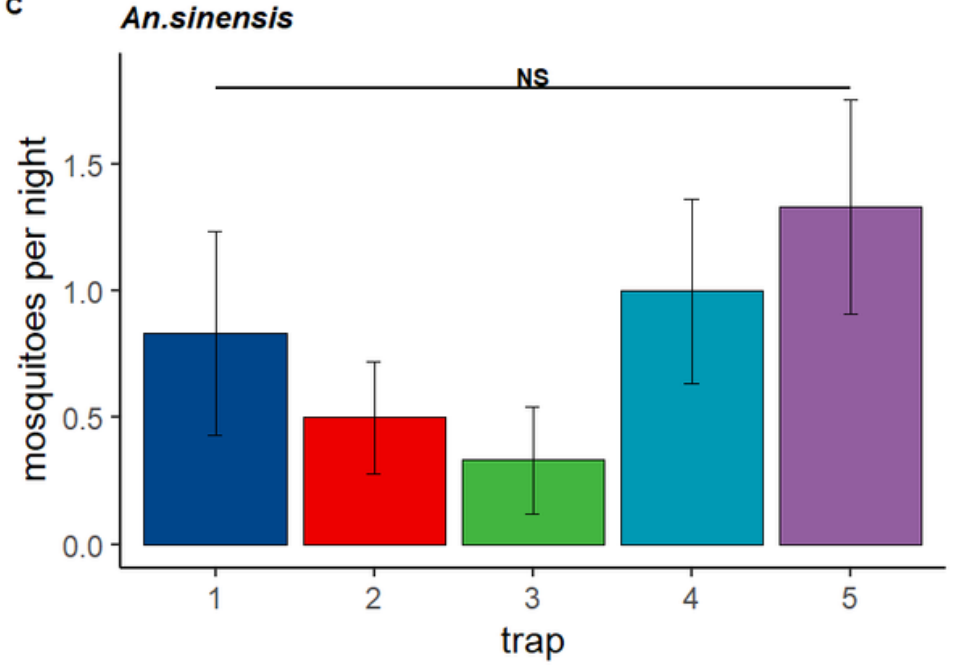

B

Cx.quinquefasciatus

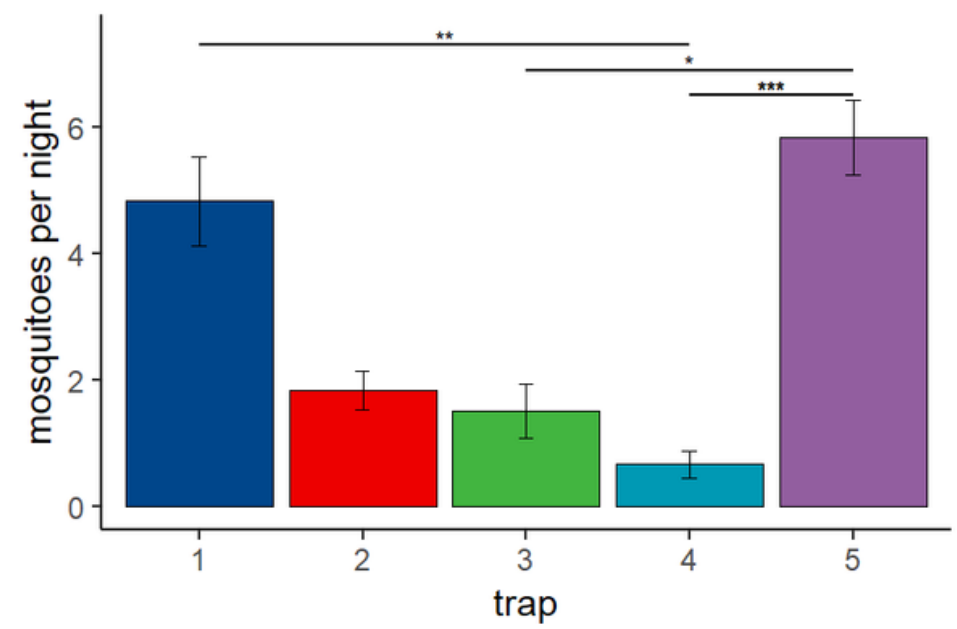

D Total

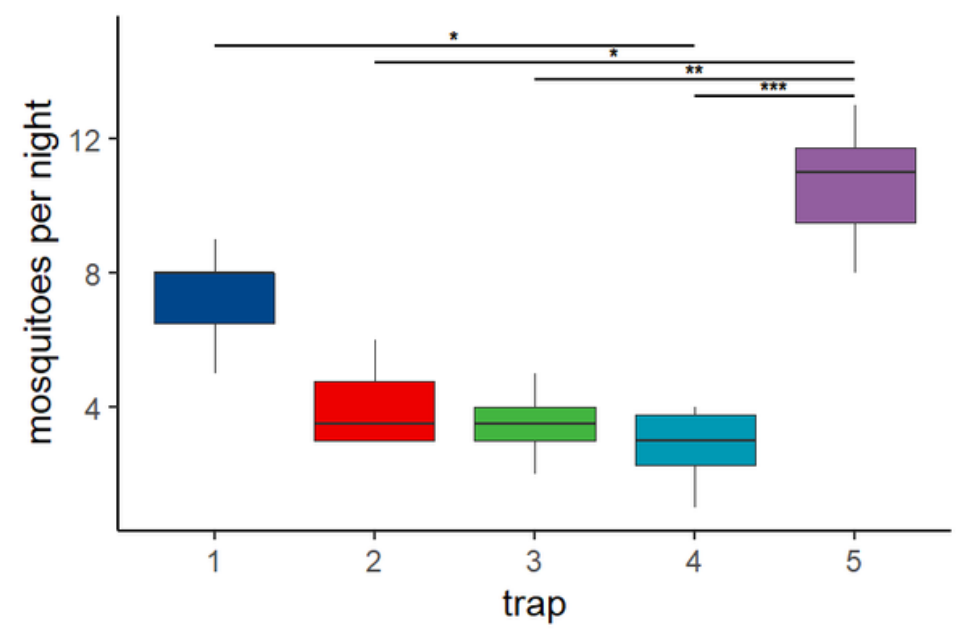

Figure 1

The number of trapped mosquitos per night among the five trapping devices. Mean +/-SE number of trapped individuals per trapping period among the five trapping devices for (A) Ae.albopictus, (B) Cx. quinquefasciatus and (C) An. sinensis.. (D)The box plots represent the median and first and third quartiles of the total species of mosquito collected in the five trap types. 

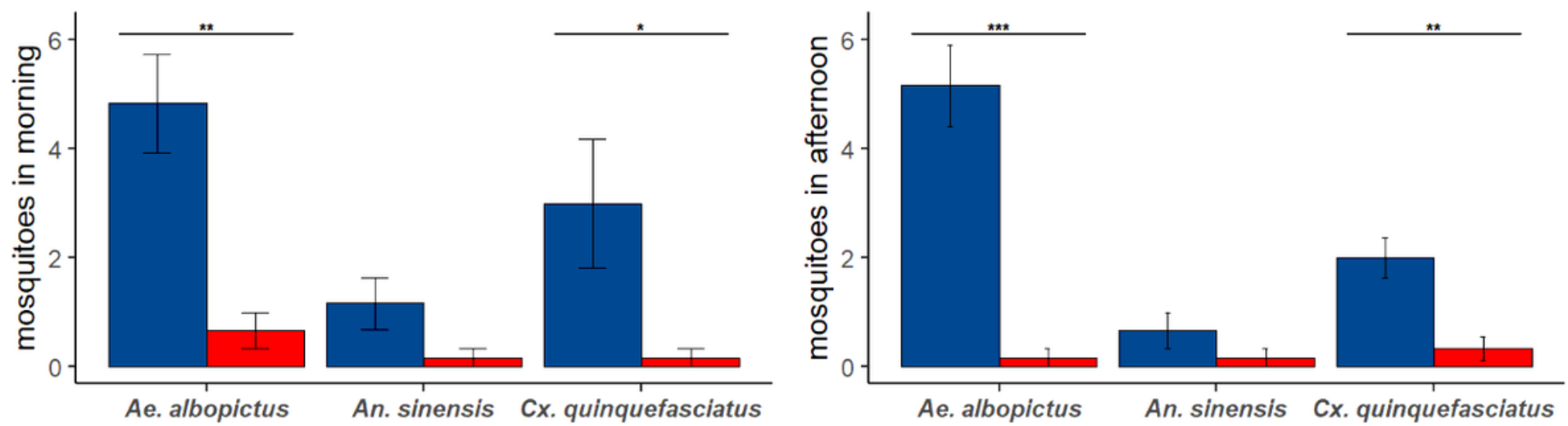

C

BGtrap $\square$ UVtrap
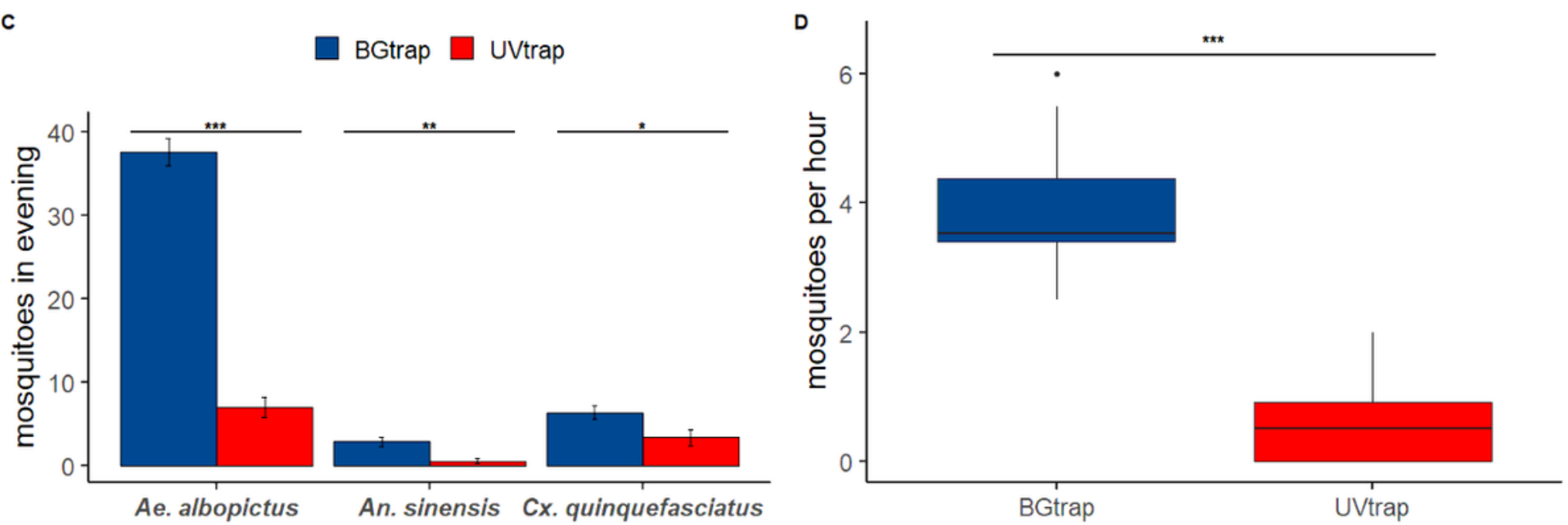

\section{Figure 2}

The number of trapped individuals per period between the two trapping devices. Mean +/-SE number of trapped individuals per trapping period between the BG-trap and UV-trap (A) in the morning, (B) afternoon, and (C) evening. (D) The box plots represent the median and first and third quartiles of the total species of mosquito collected per hour in the two trap types.

\section{Supplementary Files}

This is a list of supplementary files associated with this preprint. Click to download.

- GraphicalAbstract.docx 